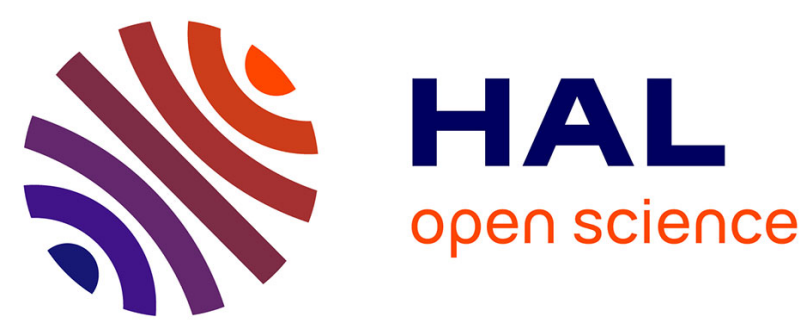

\title{
Drift driven cross-field transport and scrape-off layer width in the limit of low anomalous transport
}

Camille Baudoin, Patrick Tamain, Hugo Bufferand, Guido Ciraolo, Nicolas

Fedorczak, Davide Galassi, Alberto Gallo, Philippe Ghendrih, Nicolas Nace

\section{- To cite this version:}

Camille Baudoin, Patrick Tamain, Hugo Bufferand, Guido Ciraolo, Nicolas Fedorczak, et al.. Drift driven cross-field transport and scrape-off layer width in the limit of low anomalous transport. Plasma Physics and Controlled Fusion, 2018, 60 (10), pp.105007. 10.1088/1361-6587/aad650 . hal-02111781

\section{HAL Id: hal-02111781 \\ https://hal-amu.archives-ouvertes.fr/hal-02111781}

Submitted on 26 Apr 2019

HAL is a multi-disciplinary open access archive for the deposit and dissemination of scientific research documents, whether they are published or not. The documents may come from teaching and research institutions in France or abroad, or from public or private research centers.
L'archive ouverte pluridisciplinaire HAL, est destinée au dépôt et à la diffusion de documents scientifiques de niveau recherche, publiés ou non, émanant des établissements d'enseignement et de recherche français ou étrangers, des laboratoires publics ou privés. 
archives-ouvertes

\section{Drift driven cross-field transport and scrape-off layer width in the limit of low anomalous transport}

Camille Baudoin, Patrick Tamain, Hugo Bufferand, Guido Ciraolo, Nicolas

Fedorczak, Davide Galassi, Alberto Gallo, Philippe Ghendrih, Nicolas Nace

\section{- To cite this version:}

Camille Baudoin, Patrick Tamain, Hugo Bufferand, Guido Ciraolo, Nicolas Fedorczak, et al.. Drift driven cross-field transport and scrape-off layer width in the limit of low anomalous transport. Plasma Physics and Controlled Fusion, IOP Publishing, 2018, 60 (10), pp.105007. 10.1088/1361$6587 /$ aad650 . hal-02111781

\section{HAL Id: hal-02111781 \\ https://hal-amu.archives-ouvertes.fr/hal-02111781}

Submitted on 26 Apr 2019

HAL is a multi-disciplinary open access archive for the deposit and dissemination of scientific research documents, whether they are published or not. The documents may come from teaching and research institutions in France or abroad, or from public or private research centers.
L'archive ouverte pluridisciplinaire HAL, est destinée au dépôt et à la diffusion de documents scientifiques de niveau recherche, publiés ou non, émanant des établissements d'enseignement et de recherche français ou étrangers, des laboratoires publics ou privés. 


\title{
Drift driven cross-field transport and scrape- off layer width in the limit of low anomalous transport
}

\author{
Camille Baudoin $^{1,2}$ (1) Patrick Tamain $^{1}$, Hugo Bufferand ${ }^{1}$, Guido Ciraolo ${ }^{1}$, \\ Nicolas Fedorczak ${ }^{1}$, Davide Galassi ${ }^{2}{ }^{(1)}$, Alberto Gallo ${ }^{1}{ }^{10}$, \\ Philippe Ghendrih ${ }^{1}$ and Nicolas Nace ${ }^{1,2}$
}

${ }^{1}$ CEA, IRFM, F-13108 Saint-Paul-lez-Durance, France

${ }^{2}$ Aix Marseille Université, CNRS, Centrale Marseille, M2P2, Marseille, France

E-mail: camille.baudoin@gmail.com

Received 22 December 2017, revised 6 July 2018

Accepted for publication 27 July 2018

Published 23 August 2018

\begin{abstract}
The impact of the $\nabla B$-drift in the cross-field transport and its effect on the density and power scrapeoff layer (SOL) width in the limit of low anomalous transport is studied with the fluid code SolEdge2D. In the first part of the work, the simulations are run with an isothermal reduced fluid model. It is found that a $\nabla B$-drift dominated regime is reached in all geometries studied (JET-like, ASDEX-like and circular analytic geometries), and that the transition toward this regime comes along with the apparition of supersonic shocks, and a complex parallel equilibrium. The parametric dependencies of the SOL width in this regime are investigated, and the temperature and the poloidal magnetic field are found to be the principal parameters governing the evolution of the SOL width. In the second part of this paper, the impact of additional physics is studied (inclusion of the centrifugal drift, self-consistent variation of temperature and the treatment of the neutral species). The addition of centrifugal drift and neutral species are shown to play a role in the establishment of the parallel equilibrium, impacting the SOL's width, although the role of the centrifugal drift is limited to a low diffusion level. Finally, the numerical results are compared with the estimate of the Goldston's heuristic drift based model (HD-model), the starting point of our study, and which has shown good agreement with experimental scaling laws. We find that the particles SOL widths in the $\nabla B$-drift dominated regime are at least two times smaller than the estimate of the HD-model. Moreover, in the parametric dependencies proposed by the HD-model, the dependency with $B_{\mathrm{pol}}$ is retrieved, but not the one on $T$.
\end{abstract}

Keywords: edge plasma, SOL width, drift, heat transport

(Some figures may appear in colour only in the online journal)

\section{Introduction}

Economic viability of future fusion devices requires a sufficient power spreading on plasma facing components (PFC) that are potentially damaged by high energy deposition, for example for ITER the divertor materials constraint in steadystate is estimated at $10 \mathrm{MW} \mathrm{m}^{-2}$ [1]. For a given power crossing the separatrix $\left(P_{\mathrm{SOL}}\right)$, the peak heat load $\left(q_{\max }\right)$ is set by the scrape-off layer (SOL) heat channel width, commonly named $\lambda_{q}$, and divertor spreading $S_{q}$ [2], hence the heat channel width is a key parameter for tokamak operation.
The description of heat load at the target [2], which takes into account both transport in the main SOL and in the divertor region, has permitted the removal of geometrical effects and the extraction of a coherent multi-machine (JET, DIII-D, ASDEX Upgrade, C-Mod, NSTX, MAST) empirical scaling law for the H-mode $[3,4]$. The strongest dependency found is the one with the poloidal magnetic field $\left(B_{\mathrm{pol}}\right)$, giving $\lambda_{q \text {,Eich }} \propto B_{\text {pol }}^{-1.16}$, where $\lambda_{q \text {,Eich }}$ is the SOL width estimated at the target with Eich's method [2]. This functional dependency has been retrieved in other experiments, in MAST [5] and in $\lambda_{T_{e}}$ at the mid-plane, in agreement with Eich's scaling 
considering a two point model [6]. It is worth noting that this feature is not specific to the H-mode, an empirical scaling law on JET and ASDEX Upgrade in the L-mode shows similar dependency with $B_{\text {pol }}[7,8]$. The extrapolation of the H-mode scaling law to ITER has predicted $\lambda_{q}=1 \mathrm{~mm}$, raising concern for future reactor devices.

So far, empirical scaling laws lack a theoretical grounding when confirming their predictions, and the mechanisms driving the heat transverse transport in edge plasma are not yet fully understood. This is a central issue for the design and the sizing of future devices. Some theoretical studies attempting to describe the way energy escapes the core plasma through the separatrix and deposits on the PFC have been carried out, but no definitive conclusion has yet been drawn. In the L-mode, it is accepted that turbulence dominates the cross-field transport, and theoretical models have been proposed based on linear analysis using the gradient removal theory [9], and based on a blob approach [10], to predict the value of the SOL width in L-mode. In the H-mode turbulence is strongly reduced in the pedestal and near SOL. It is thus not clear which is the main mechanism driving the transport through the separatrix. A heuristic drift based (HD) model [11] proposed that the curvature drift is the main mechanism driving the cross-field transport across the separatrix in the H-mode determining entirely the SOL power width. A similar description of the edge transport was earlier proposed by [12] with a neoclassical approach. This model has attracted much interest for its good agreement with experience [3, 13, 14], in particular its prediction of the dependency on $B_{\text {pol }}$.

The work presented in this paper aims to understand the convective transport via the curvature drifts, in the limit of weak collisional and anomalous transport. The understanding of the HD-model is the underlying motivation of this work, thus the fluid model used here includes restrictive assumptions taking into account the heuristics of the HD-model, except for the assumption on the parallel equilibrium, selfconsistently solved in the simulations. Our numerical results allow us to question two things: the internal consistency of the model, and its consistency with experimental observations. Similar work has been published [15] assessing the existence of a regime where the SOL width is determined by $\nabla B$-drift. In this study, we use a different numerical model, the 2D fluid code SolEdge2D, to further investigate the framework of such a $\nabla B$-drift dominated regime: its characteristics, its dependencies on parameters and on geometry, and its limits. In particular, we study the impact of the addition of the centrifugal drift, the inclusion of temperature variation and of the neutral species. Previously published work [16], closely related to this work, has studied a more complete drift based transport in a higher level anomalous transport, in particular pointing out the importance of the electric drift in cross-field drift transport. The study of electric drift transport is out of the scope of this paper, and the full drift dynamics should be discussed in future work.

We first seek a regime where the level of turbulence, set via a diffusion coefficient in the model, does not impact the SOL width. Even in a low turbulence regime, it is not obvious that the magnetic drift could contribute significantly to the flux-surface averaged transport as the drift presents an up/ down symmetry. Hence, an outward flux-surface transport would require a strong up/down asymmetry [17]. We then characterize such a regime and finally compare the SOL width to the estimate of the heuristic model.

After a brief description of the SolEdge2D model (section 2), the impact of the $\nabla B$-drift on cross-field transport, plasma parallel equilibriums and radial profiles is first studied at different levels of anomalous transport in a JET-like geometry (section 3). After establishing the existence of a $\nabla B$-drift dominated regime, we estimate SOL widths found in such a regime. We also look at the parametric functional dependencies (section 3.4) and the impact of geometry on the SOL width (section 4). In section 5, we study the impact of the centrifugal drift, another component of the magnetic drift not always taken into account in fluid models. In section 6 , we release the isothermal assumption, and we study the impact of the inclusion of neutral species. Finally, in section 7, we draw a comparison between of numerical results and the estimate of the HD-model for the SOL width, and we discuss the assumptions made in the model.

\section{Model description: setup and geometry}

In this section, the model used for this study is briefly described, see [18] for more details. SolEdge2D is a mean field transport code that assumes toroidal axisymmetry of all field and solves drift-reduced fluid equations with Braginskii closure for electrons and an arbitrary number of ion species. In the single ion case, of interest in this paper, the model solves, for the ion density $n$, parallel ion velocity $u_{\|}$, and temperature of electrons and ions $T_{e}$ and $T_{i}$, equations (1)-(4), which are solved using a finite volume numerical scheme. Note that in sections $3-5$, we use an isothermal model, solving only equations (1)-(2) with $T_{e}=T_{i}=$ constant, and the temperature variations are only included in section 6 . At the scale of the mesh grid, larger than the Debye length, the quasi-neutrality is justified, so that the electron $\left(n_{e}\right)$ and ion $\left(n_{i}\right)$ densities are taken equal $n=n_{e}=n_{i}$. Moreover, in this work, we do not consider the physics of charge balance, thus we assume ambipolarity for parallel current $j_{\|}=0$ so that electron and ion parallel velocity are supposed to be equal so that $u_{\|}=u_{\|, e}=u_{\|, i}$. This is an important assumption since the $\nabla B$-drift is charge dependent and induces a plasma polarization which should be discussed. This is the first restrictive assumption of the studied model, this is a deliberate choice made to follow the framework of the HD-model which was the starting point of our study.

$$
\partial_{t} n+\nabla \cdot\left(n u_{\|} \mathbf{b}+n \mathbf{u}_{\perp}\right)=S^{n}
$$

$\partial_{t}\left(n u_{\|}\right)+\nabla \cdot\left(n u_{\|}\left(u_{\|} \mathbf{b}+\mathbf{u}_{\perp}\right)\right)=-\nabla_{\|} \frac{p_{i}}{m_{i}}+\frac{e n E_{\|}}{m_{i}}+\frac{R_{e i}}{m_{i}}$

$+\nabla \cdot\left(\nu_{\perp} n \nabla_{\perp} u_{\|}\right)+S^{n u}$ 
(a) JET

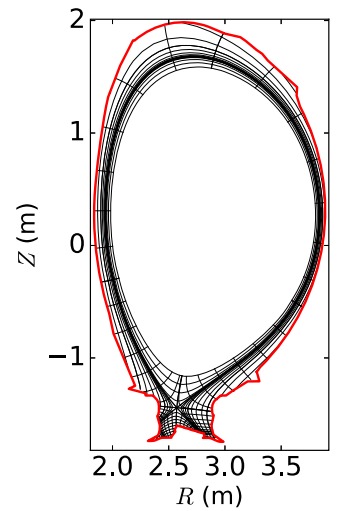

(b) COMPASS

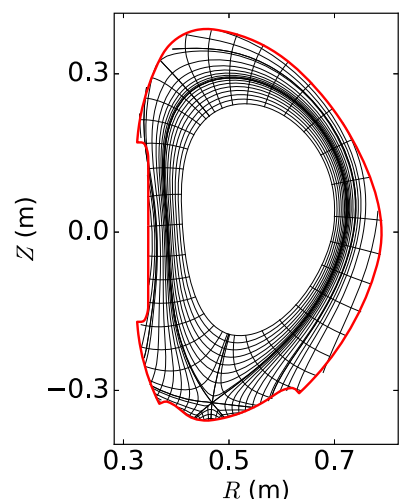

(c) limiter

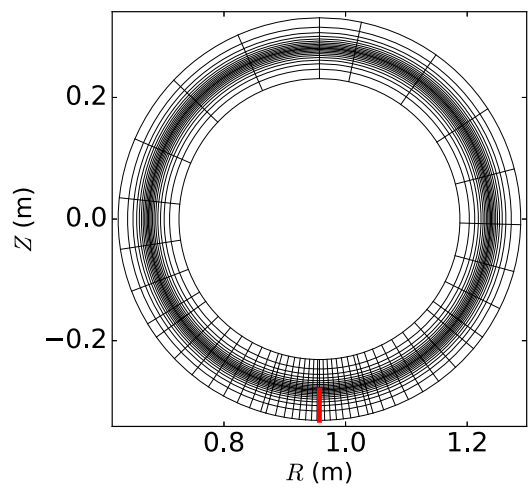

Figure 1. Sketch of computational grid (black lines) for the diverted JET-like (a) and COMPASS-like (b) plasma equilibriums with the wall position (red lines), (c) for the analytical circular equilibrium with the limiter position (red lines); for the purpose of legibility only one in three edges are reported for the JET-like grid and one in four for circular geometry.

$$
\begin{aligned}
& \partial_{t}\left(\frac{3}{2} n T_{i}+\frac{1}{2} m_{i} n u_{\|}^{2}\right)+\nabla \cdot\left(\left(\frac{5}{2} n T_{i}+\frac{1}{2} m_{i} n u_{\|}^{2}\right)\left(u_{\|} \mathbf{b}+\mathbf{u}_{\perp}\right)\right) \\
& =\nabla \cdot\left(\kappa_{i} \nabla_{\|} T_{i} \mathbf{b}+\chi_{\perp, i} n \nabla_{\perp} T_{i}+\nu_{\perp} n \nabla_{\perp}\left(\frac{1}{2} m_{i} u_{\|}^{2}\right)\right) \\
& \quad+e n u_{\|} E_{\|}+u_{\|} R_{e i}+Q_{e i}+S^{E_{i}},
\end{aligned}
$$$$
\begin{gathered}
\partial_{t}\left(\frac{3}{2} n T_{e}\right)+\nabla \cdot\left(\frac{5}{2} n T_{e}\left(u_{\|} \mathbf{b}+\mathbf{u}_{\perp}\right)\right) \\
\quad=\nabla \cdot\left(\kappa_{e} \nabla_{\|} T_{e} \mathbf{b}+\chi_{\perp, e} n \nabla_{\perp} T_{e}\right) \\
-e n u_{\|} E_{\|}-u_{\|} R_{e i}-Q_{e i}+S^{E_{e}}
\end{gathered}
$$

where $\mathbf{b}$ denotes the direction of the magnetic field $\mathbf{b}=\frac{\mathbf{B}}{B}, m_{i}$ is the ion mass, $e$ is the elementary charge, $\kappa_{i}, \kappa_{e}$ are respectively the coefficients of parallel conduction for electrons and ions, and $Q_{e i}$ is an energy transfer due to collision. The inertia and viscosity terms of electrons are neglected giving $e n E_{\|}=-\nabla_{\|}\left(p_{e}\right)-R_{e i}$ where $E_{\|}$is the parallel electric field and $R_{e i}=0.71 n \nabla_{\|} T_{e}-\eta_{\|} n j_{\|}$is the parallel friction force. Here, $R_{e i}$ is simplified to $R_{e i}=0.71 n \nabla_{\|} T_{e}$ under the assumption $j_{\|}=0$. The terms $S^{n}, S^{n u}, S^{E_{e}}$ and $S^{E_{i}}$ are external sources or losses due to collisions/reactions with other species for density, momentum, and electron and ion energy, they are thus null in the case where neutral species are not included. In standard 2D transport code, turbulence is inhibited as turbulence requires the treatment of the two transverse directions, and the cross-field anomalous transport is arbitrarily set via diffusion operator. The cross-field flux is then equal to $n \mathbf{u}_{\perp}=-D_{\perp} \nabla_{\perp} n+n \mathbf{u}_{\text {drift }}$. The diffusion coefficient $D_{\perp}$ stands for the local level of anomalous transverse transport. For all the following simulations, $D_{\perp}$ is set to $1 \mathrm{~m}^{2} \mathrm{~s}^{-1}$ on a zone of few millimeters at the inner and outer borders to avoid edge effect, and is equal to a constant, labeled $D$, on the remaining part of the grid. $D$ is the main free parameter of this work and will be scanned to study the impact of the fluid drifts on the transverse transport for different levels of anomalous transport. The viscosity $\nu_{\perp}$ is equal to the diffusivity $D_{\perp}$ on all the domain, and ion and electron thermal diffusivities $\left(\chi_{\perp, i}, \chi_{\perp, e}\right)$ are constant on all the domain (equal to $1 \mathrm{~m}^{2} \mathrm{~s}^{-1}$ or $0.1 \mathrm{~m}^{2} \mathrm{~s}^{-1}$ ). In this work, only the magnetic drift is considered, $\mathbf{u}_{\text {drift }}=\mathbf{u}_{\nabla B, i}+\mathbf{u}_{c}$, where $\mathbf{u}_{\nabla B}$ (equation (5)) denotes the $\nabla B$-drift (in the fluid approach $n \mathbf{u}_{\nabla B}$ divergence coincides with the divergence of the diamagnetic drift flux), and $\mathbf{u}_{\text {cent }}$ (equation (6)) denotes the centrifugal drift [19]. The magnetic field is in the 'normal' direction, that is to say $\mathbf{u}_{\nabla B}$ is directed downward for ions. Such drift expression does not follow a coherent drift ordering at first order detailed in the paper [20]. Indeed, the electric drift is omitted in spite of the fact that this is a drift of first order. This is the second restrictive assumption of the studied model. Once again this is a deliberate choice following the heuristics of the HD-model. The implications of such an assumption are discussed in the conclusion and are also detailed in appendix A of [15].

$$
\begin{gathered}
\mathbf{u}_{\nabla B, i}=\frac{\mathbf{B} \times \nabla p_{i}}{q B^{2}}, \\
\mathbf{u}_{\text {cent }}=\frac{m v_{\|}^{2}}{q B^{2}} \mathbf{B} \times \mathbf{b} \cdot \nabla \mathbf{b} .
\end{gathered}
$$

Three set of simulations are considered: (1) simulations including no drifts, referred as diffusion simulations, (2) simulations with including only $\nabla B$-drift, and (3) simulations including both $\nabla B$ and centrifugal drifts. In the first stage, sections $3-5$, the plasma is assumed isothermal, and $T$ is constant on all the domain, with $T_{e}=T_{i}=50 \mathrm{eV}$, and neutrals are not taken into account. These assumptions are relaxed in section 6 to evaluate how they impact the transport and plasma equilibriums.

The simulated domain extends from closed flux surfaces in the vicinity of the separatrix, with Dirichlet condition imposed to density $n=e-19 \mathrm{~m}^{-3}$ at the core boundary, to open flux surfaces up to the wall. In the parallel direction Bohm boundary conditions are imposed at the sheath entrance. Simulations have been run for three magnetic equilibriums, as illustrated in figure 1(a), a realistic diverted JET-like geometry on a $80 \times 139(r, \theta)$-grid with the following parameters $R_{0}=2.895 \mathrm{~m}, a=0.950 \mathrm{~m}$, the safety 

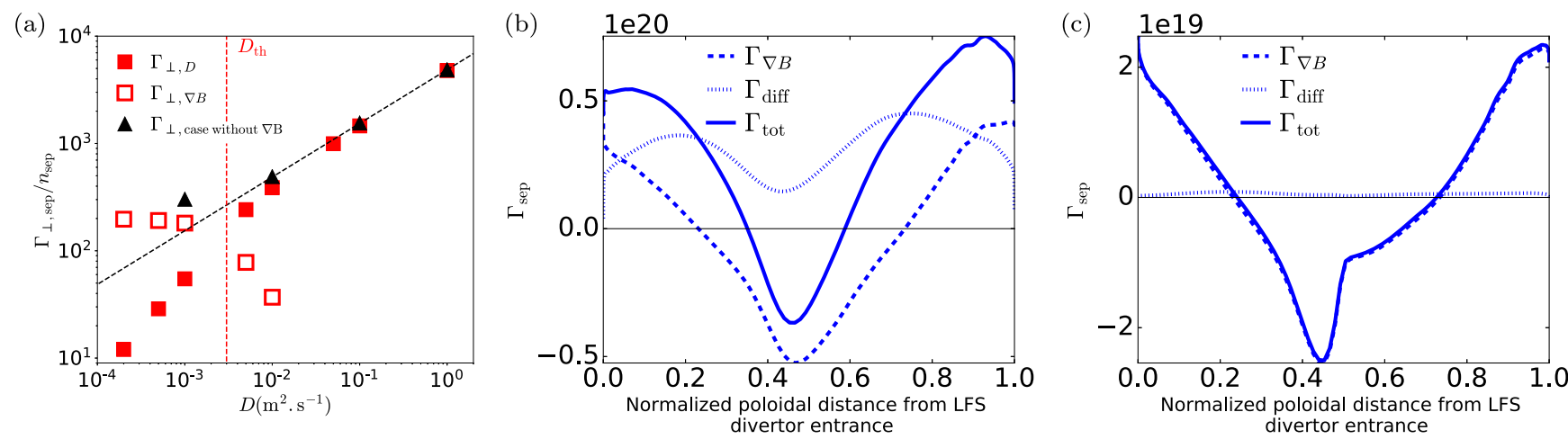

Figure 2. (a) Total particle fluxes through the separatrix normalized by the mean density due to diffusion process (red full squares), to $\nabla B$-drift (red empty squares) for $\nabla B$-drift simulations, and for diffusive simulations (black triangles) as a function of $D$. (b), (c) Poloidal distribution of the particle flux: global (full line), by diffusion (dot-dashed line), by $\mathbf{u}_{\nabla B}$ (dashed line) for $D=1 \times 10^{-1} \mathrm{~m}^{2} \mathrm{~s}^{-1}$ (b) and $D=1 \times 10^{-3} \mathrm{~m}^{2} \mathrm{~s}^{-1}(\mathrm{c})$.

factor $q_{95}$, defined as the safety factor on the flux-surface $\psi$ such as $\psi / \psi_{\text {sep }}=0.95$, is about 5 in the near SOL and $B_{0}=2.2 \mathrm{~T}$, (b) a realistic diverted COMPASS-like geometry on a $48 \times 139(r, \theta)$-grid with the following parameters $R_{0}=0.55 \mathrm{~m}, a=0.17 \mathrm{~m}, q_{95} \approx 4, B_{0}=1.15 \mathrm{~T}$, (c) an analytical circular geometry on a $240 \times 180(r, \theta)$-grid, with the following parameters $R_{0}=1,075 \mathrm{~m}, \quad a=0.287 \mathrm{~m}$, $q_{95} \approx 4, B_{0}=1 \mathrm{~T}$. On a diverted equilibrium, a penalization technique, a numerical technique to ensure Bohm-Chodura conditions at the wall detailed in [18], is used which allows simulation of the plasma up to the first wall to be enabled, as shown with a red line on figures 1(a) and (b).

\section{Convective transport by $u_{\nabla B}$ at low anomalous transport in JET-like geometry}

\section{1. $\nabla B$-drift dominated regime in the limit of low anomalous transport}

To study the role played by the $\nabla B$-drift in the particle transverse transport, two sets of JET-like isothermal simulations are first considered, the diffusive case, and the case including only the $\nabla B$-drift, for various levels of $D$. In these sets of simulations the coefficient $D$ is scanned between $1 \mathrm{~m}^{2} \mathrm{~s}^{-1}$ and $2 \times 10^{-4} \mathrm{~m}^{2} \mathrm{~s}^{-1}$, with the aim to find out whether a regime where the large scale convection by $\mathbf{u}_{\nabla B}$ is the main mechanism of particle transverse transport exists and what would be the properties of such a plasma equilibrium.

On figure 2(a) total particle flux through the separatrix due to the diffusion process equation (7) and due to the convection via $\mathbf{u}_{\nabla B}$ equation (8) are reported for both $\nabla B$-drift and purely diffusive simulations as a function of $D$. Note that all fluxes are normalized by the mean density at the separatrix.

$$
\begin{gathered}
\Gamma_{\perp, D}=\int_{\text {sep }}-D_{\perp} \nabla_{\perp} n \cdot \nabla \psi \\
\Gamma_{\perp, \nabla B}=\int_{\text {sep }} n \mathbf{u}_{\nabla B} \cdot \nabla \psi
\end{gathered}
$$

For $D \geqslant 1 \times 10^{-2} \mathrm{~m}^{2} \mathrm{~s}^{-1}$, the diffusive operator is responsible for all the transport and the averaged convective $\mathbf{u}_{\nabla B}$ flux is even slightly negative. In that range of $D$, there is no significant difference of $\Gamma_{\perp, n}$ between simulations with or without drift, in both cases the particle flux scales as $\sqrt{D}$, expected for a standard diffusive process. At $D=$ $1 \times 10^{-2} \mathrm{~m}^{2} \mathrm{~s}^{-1}$, the $\mathbf{u}_{\nabla B}$ convective contribution in the particle balance starts to rise and its relative weight continues to rise with the decreasing level of transverse transport to end up largely dominant for $D \leqslant 1 \times 10^{-3} \mathrm{~m}^{2} \mathrm{~s}^{-1}$, with a contribution of more than $90 \%$ in the total balance. Hence, a $\nabla B$-drift dominated regime is reached at low transverse transport. In the following part of the paper, we denote as $D_{\text {th }}$ the value of $D$ at the transition toward the $\nabla B$ dominated regime, that is to say the diffusion value below which the $\nabla B$-drift starts to be the major player in the transverse transport, i.e. when $\Gamma_{\perp, \nabla B} \sim \Gamma_{\perp \text {,diff }}$. For this set of simulations (isothermal with JET-like geometry), we have $1 \times 10^{-3}<$ $D_{\text {th }}<5 \times 10^{-3}$. At this level of $D$, the diffusion source in the $\nabla B$-drift simulations decreases faster than for the purely diffusive simulations due to a relative flattening of the radial profile around the separatrix (see section 3.3).

To give an indication of what would represent such a level of transverse transport, let us recall some typical value of diffusion coefficients used when addressing anomalous transverse transport, as well as classical and neoclassical processes. The typical diffusion for anomalous transport used in the pedestal to match profiles in H-mode plasmas is of the order of $1 \times 10^{-1} \mathrm{~m}^{2} \mathrm{~s}^{-1}$ [21-24]; the estimation of diffusion representing the dissipative process as established in [25] gives a typical diffusion of $D_{\mathrm{C}} \approx 1.5 \times 10^{-3} \mathrm{~m}^{2} \mathrm{~s}^{-1}$ for the classical process and $D_{\mathrm{NC}} \approx 2.5 \times 10^{-2} \mathrm{~m}^{2} \mathrm{~s}^{-1}$ for the neoclassical process. Thus, the transition toward a drift dominated regime appears to take place well below the neoclassical level, and $\mathbf{u}_{\nabla B}$ is predominant only at a diffusion level lower than the classical level, which raises the question of the possibility of achieving such a regime.

It is important to stress that the appearance of a flux-surface averaged particle transport by $\mathbf{u}_{\nabla B}$ is inevitably associated with strong poloidal asymmetries. Indeed, $\Gamma_{\nabla B}=\int_{\text {sep }} n \mathbf{u}_{\nabla B} \cdot \nabla \psi$ 

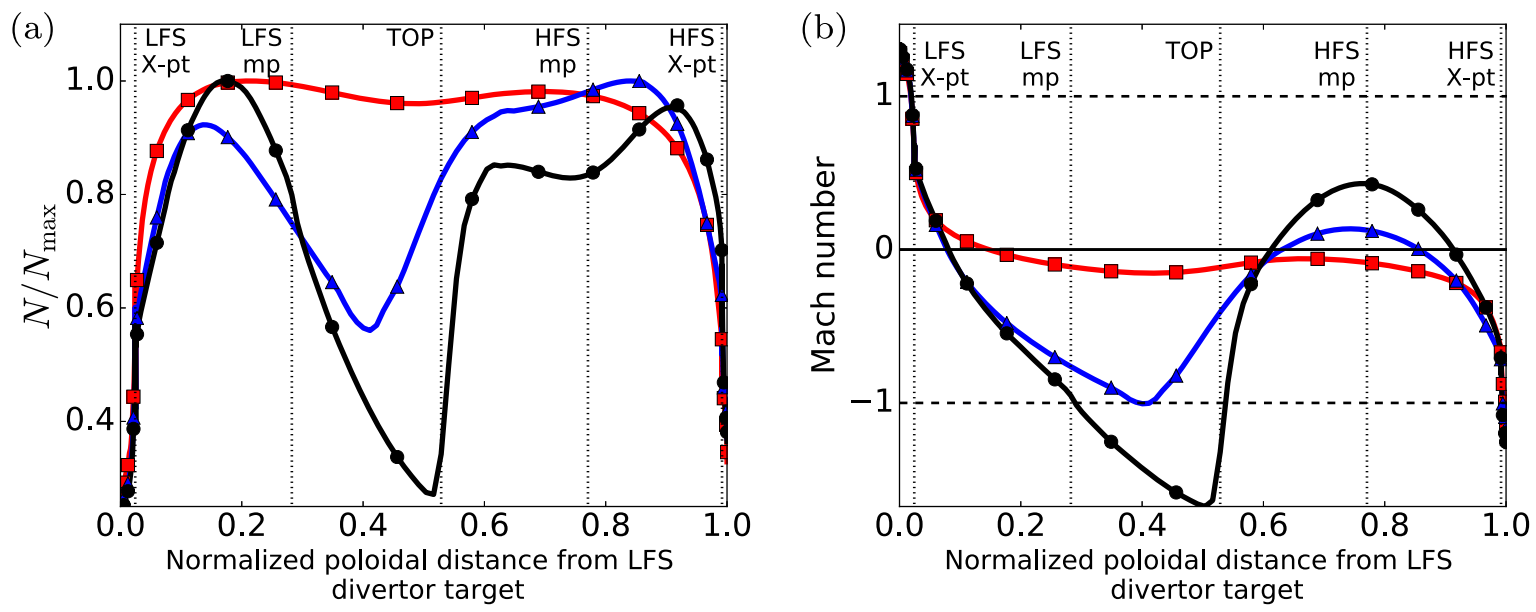

Figure 3. Poloidal profile in the near SOL $\left(\frac{r}{a}=1.004\right)$ of the normalized density (a), and the parallel Mach number (b) for $\nabla B$-drift simulations for $D=1 \times 10^{-1} \mathrm{~m}^{2} \mathrm{~s}^{-1}$ (red line with squares), $D=5 \times 10^{-3} \mathrm{~m}^{2} \mathrm{~s}^{-1}$ (blue line with triangles), and $D=2 \times 10^{-4} \mathrm{~m}^{2} \mathrm{~s}^{-1}$ (black line with circles).

yet $\int_{\text {sep }} \mathbf{u}_{\nabla B} \cdot \nabla \psi=0$ due to the vertical symmetry of the $\nabla B$-drift implying that a sufficient up/down asymmetry is necessary to permit $\Gamma_{\nabla B}$ to be positive. Figures 2(b) and (c) show the poloidal local flux, where at relatively high diffusion $D=0.1 \mathrm{~m}^{2} \mathrm{~s}^{-1}, \mathbf{u}_{\nabla B}$ convection is found to contribute significantly locally, but with the symmetry the positive flux at the bottom strictly compensates the sink at the top, so the fluxsurface contribution is negligible. At low anomalous transport, figure 2(c), the $\mathbf{u}_{\nabla B}$ convective flux exhibits an up-down asymmetry, and the source at the bottom of the torus becomes bigger than the sink at the top, explaining the rise of its weight in the flux-surface balance.

\subsection{Equilibrium in drift dominated regime}

In the previous section, it has been shown that at $D<D_{\text {th }}$ with $1 \times 10^{-3} \mathrm{~m}^{2} \mathrm{~s}^{-1}<D_{\text {th }}<5 \times 10^{-3} \mathrm{~m}^{2} \mathrm{~s}^{-1}$ the convection by $\mathbf{u}_{\nabla B}$ is the predominant particle source for the SOL, at both local and global scales. Let us now characterize the plasma equilibrium in such a regime. On figure 3 , the poloidal profiles in the near SOL of density (a) and Mach number $M=\frac{\sqrt{m_{i}} u_{\|}}{\sqrt{T_{e}+T_{i}}}$ (b) are reported. At $D>2 \times 10^{-2}$ density is almost constant in the poloidal direction, and the Mach number is relatively low far from the target. At low $D$, the most striking result is the appearance of a complex parallel equilibrium presenting a supersonic transition that comes along with the rise of sharp gradients and strong asymmetry of the density in the poloidal direction. This is a surprising result as in standard fashion it is considered that the flows stay subsonic in the edge plasma. If recent works underline the possible existence of supersonic flows close to the target $[26,27]$, this is not the case for the main SOL where the flows are believed to be subsonic. Moreover, such flows come also with high asymmetry of density up to a factor of four, once again such characteristics are surprising as they have not been observed so far in experiments. Note that the density tends to be higher at the bottom of the torus, in agreement with the direction of the $\mathbf{u}_{\nabla B}$ convection, which leads to an effective particle source. This supersonic transition at low $D$ comes from the strong asymmetry of the effective particle source, composed of a large source at the bottom of the torus and a large sink at the top, creating an important Pfirsch-Schlüter return flow. When decreasing the diffusion coefficient, the pressure gradient increases, and it enlarges the PfirschSchlüter flow's amplitude $\left(\Gamma_{\|, P S} \propto \nabla P\right)$, reaching a supersonic transition coming along with a steady-state shock in the poloidal direction. It has been verified that the transition is in agreement with the model of supersonic transition presented in [26].

In fact this transition can be explained by a reduced model for the parallel equilibrium equation (9). In a steadystate equilibrium, the continuity equation and the momentum balance can be simplified in equation (9) by assuming that: (1) the parallel particle flux distribution is of the form $S_{1}+S_{2} \cos \left(2 \pi z_{\|}\right)$, where $z_{\|}$is the normalized parallel coordinate $z_{\|} \in[0,1]$, representing a constant diffusive flux and a sinusoidal $\mathbf{u}_{\nabla B}$ flux in a circular geometry, (2) the total pressure is uniform in the parallel direction (i.e $n T\left(1+M^{2}\right)$ is conserved), (3) the Mach number is equal to 1 at both targets.

$$
\left\{\begin{array}{l}
\partial_{z \|} \Gamma_{\|}=S_{1}+S_{2} \cos \left(2 \pi z_{\|}\right) \\
\partial_{z \|}\left(\frac{\Gamma_{\|}^{2}}{n}+n\right)=0 \text { with } \Gamma_{\|}=n u_{\|} . \\
|M|=1 \text { for } z_{\|}=0,1
\end{array}\right.
$$

This system has the following analytical solution:

$$
\left\{\begin{array}{l}
\Gamma_{\|}\left(z_{\|}\right)=S_{1}\left(z_{\|}-0.5\right)+\frac{S_{2}}{2 \pi} \sin \left(2 \pi z_{\|}\right) \\
n\left(z_{\|}\right)=\frac{S_{1} \pm \sqrt{\Delta\left(z_{\|}\right)}}{2} \\
M\left(z_{\|}\right)=\frac{S_{1} \pm \sqrt{\Delta\left(z_{\|}\right)}}{2 \Gamma\left(z_{\|}\right)} \\
\text {with: } \Delta\left(z_{\|}\right)=S_{1}^{2}-4 \Gamma_{\|}\left(z_{\|}\right)^{2}
\end{array} .\right.
$$



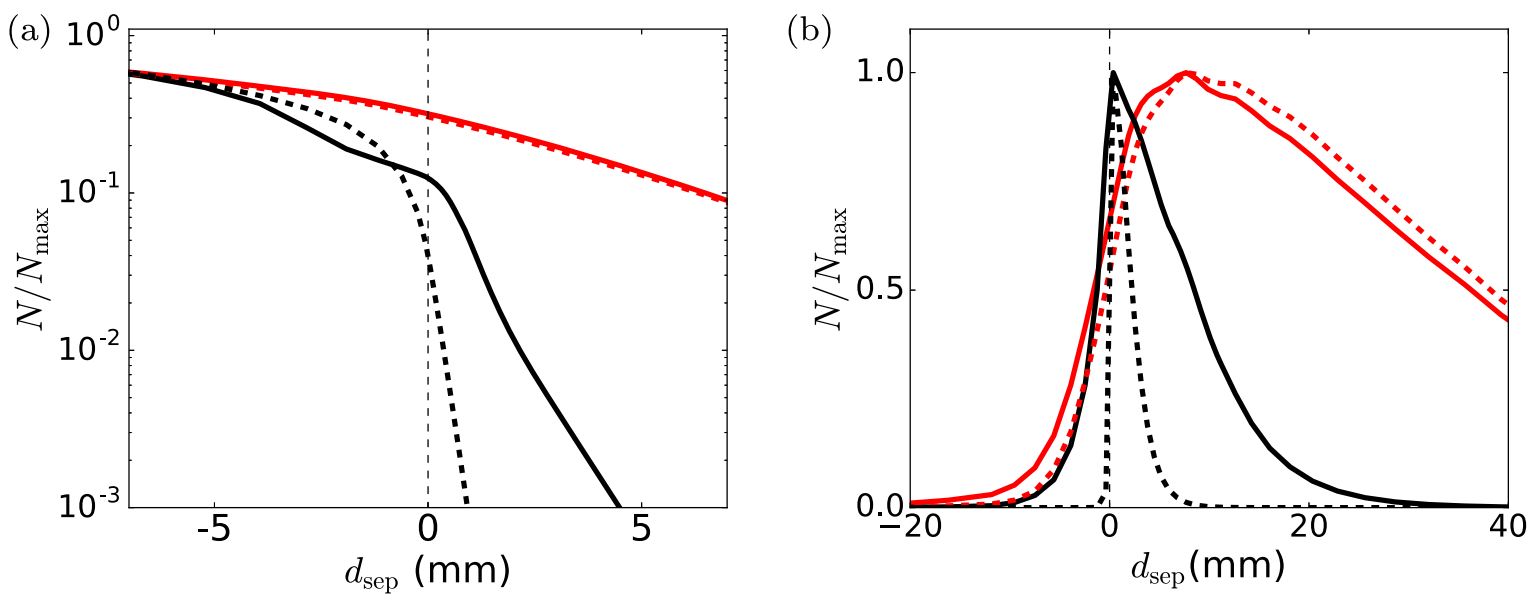

Figure 4. Density radial profile for diffusive (dashed lines) and $\nabla B$-drift (full lines) simulations at the outer mid-plane (a), and at the outer target (b), for $D=1 \times 10^{-1} \mathrm{~m}^{2} \mathrm{~s}^{-1}$ (red), and $D=2 \times 10^{-4} \mathrm{~m}^{2} \mathrm{~s}^{-1}$ (black).

When $\Delta \geqslant 0$, there always exists a subsonic smooth solution with the branch $n\left(z_{\|}\right)=\frac{S_{1}+\sqrt{\Delta\left(z_{\|}\right)}}{2}$ and $M\left(z_{\|}\right)=$ $\frac{S_{1}-\sqrt{\Delta(z \|)}}{2 \Gamma(z \mid)}$. However, when $\Delta<0$ i.e $\frac{S_{2}}{S_{1}}>4.6$ no subsonic smooth solution exists, thus it allows us to predict the threshold in $D$ when the supersonic bifurcation first appears. Let us now estimate the value of diffusion where the supersonic transition should occur. The diffusive particle flux is equal to $D \nabla_{\perp} n=D \frac{n}{\lambda_{n}}$, with the particles' SOL width, $\lambda_{n}$, equal to $\sqrt{\frac{2 \pi q R_{0} D}{c_{s}}}$ for a standard diffusive process. For the contribution of the $\nabla B$-drift, the coefficient $S_{2}$ corresponds to the maximum flux by diamagnetic drift $S_{2}=n \mathbf{u}_{\nabla B \text {, max }}$, note that $\mathbf{u}_{\nabla B}$ in the isothermal case is only a function of the geometry as the temperature is imposed. Thus, the bifurcation toward a supersonic transition should appear when $\frac{\mathbf{u}_{\nabla B}}{\frac{D}{\lambda_{n}}}=\frac{\sqrt{2 \pi q R_{0}} \mathbf{u}_{\nabla B}}{\sqrt{c_{s} D}}=4$.6. The numerical application gives $D_{\text {trans }} \approx 1.5 \times 10^{-2} \mathrm{~m}^{2} \mathrm{~s}^{-1}$ for a JET-like configuration. In simulations, supersonic flows first occur at $D=5 \times$ $10^{-3} \mathrm{~m}^{2} \mathrm{~s}^{-1}$, that it to say at lower diffusion than predicted. This discrepancy can be explained by the assumption of a circular geometry in our model equation (9). Moreover, the numerical scheme order was shown to impact the value of the transition through its ability or not to capture shocks accurately. In fact for a circular geometry with a second order weighted essentially non-oscillatory (WENO) scheme the model predict the correct value of $D_{\text {trans }}$. It is worth noting that the rise of the $\mathbf{u}_{\nabla B}$ convective transport appears at the same diffusion as supersonic transition, therefore it seems to be a necessary condition to be in a drift dominated regime of transport as it allows strong symmetry breaking of the density.

\subsection{SOL width and scaling}

We now aim to define an SOL width looking at the radial profiles in figure 4 at the outer mid-plane (a) and at the outer target (b). For $D \geqslant D_{\text {th }}$, radial profiles follow the classical description of a decreasing exponential. Profiles of both set of simulations, with or without $\mathbf{u}_{\nabla B}$, coincide except for a small inward shift of the peak at the outer target due to the inward direction of $\mathbf{u}_{\nabla B}$ below the X-point. However, for simulations with supersonic transitions, profiles of simulations with and without drift differ significantly. Moreover, profiles at the outer mid-plane of $\nabla B$-drift simulation are not well fitted by an exponential function, thus the definition of SOL width by a single e-folding length is no longer relevant. Hence, we use the so-called integral decay length on density profiles defined by $\lambda_{\mathrm{n}, \text { int }}=\int n(r) \mathrm{d} r / n_{\max }$. This is a convenient definition as it permits comparing exponential profiles with more complex ones, furthermore it is meaningful as it is directly linked to the peak value. Another way to define the SOL's width, most commonly used in experiments [3], is to proceed with an Eich's fit [2], a convolution of a decreasing exponential and a Gaussian at the outer target profile, and the resulting estimation of the SOL width is denoted $\lambda_{n \text {.Eich }}$. Since it is the most commonly used in experiments and that the SOL width described by the HD-model is the one at the target, the Eich's definition is prioritized in this section. Nevertheless, there are three cases when the Eich's definition can not be used: (1) when the profiles at the target are not well fitted by an Eich function, i.e. with a correlation coefficient below 0.95 (sections 5 and 6), (2) when we consider limiter geometry (section 4), (3) when we look at the profiles far from the wall (for the variation of the SOL width in the poloidal direction), in such cases we will use the integral description instead of Eich's. Note that all estimates of SOL width take into account the flux expansion by remapping profiles at the outer midplane in order to get rid of obvious geometric effects.

It is also important to point out that gradients vary not only in the radial direction but also in the poloidal direction. In figure 5 we observe that at low diffusion $\lambda_{n \text {,int }}$ is uniform for purely diffusive simulations, but presents variation in the poloidal direction in the drift dominated regime. Note that here variations are moderate but in other sets of simulations (see section 5) poloidal variation amplitude of $\lambda_{n, \text { int }}$ can reach a factor of four. In conclusion, one must keep in mind that an estimation of an SOL width by a single value summarizes the transverse transport but is an oversimplification and hides the complexity of the plasma equilibrium. 


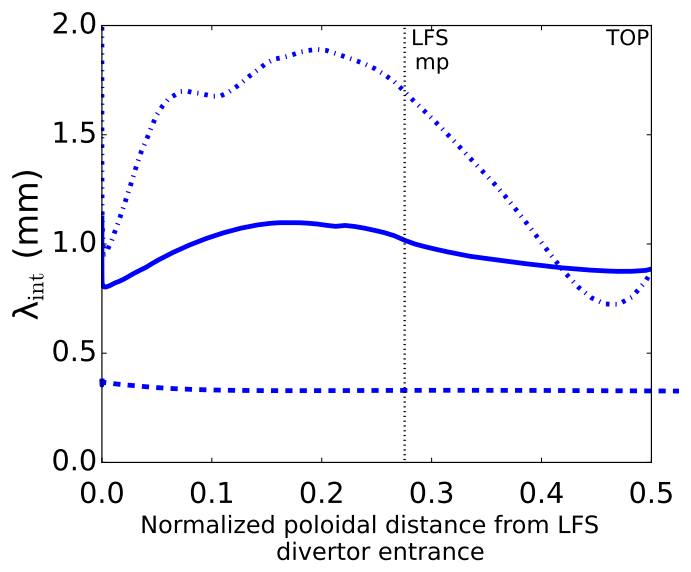

Figure 5. Poloidal profile of $\lambda_{n \text {,int }}$ for diffusive (dashed line), $\nabla B$-drift (full line) and both $\nabla B$ and centrifugal drift (dot-dashed line) simulations for $D=2 \times 10^{-4} \mathrm{~m}^{2} \mathrm{~s}^{-1}$.

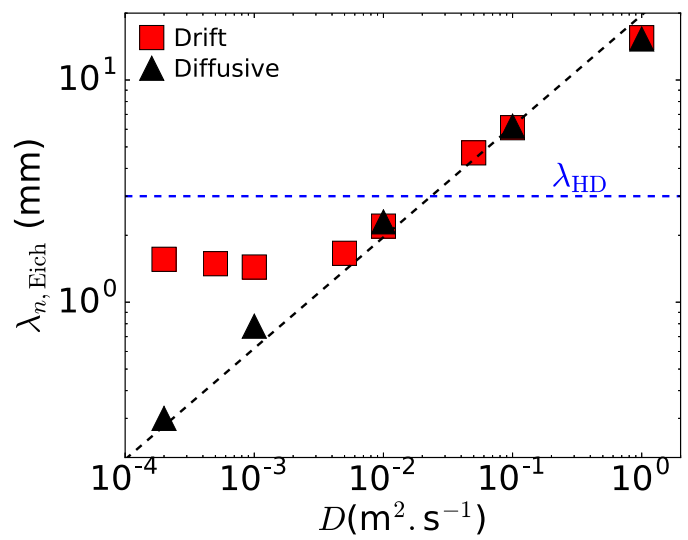

Figure 6. $\lambda_{n \text {,Eich }}$ for purely diffusive (black triangles), and for $\nabla B$-drift (red squares) simulations as a function of the diffusion coefficient, the dashed straight lines represents the $\sqrt{D}$ scaling, and the $x$ - and $y$-axis are in log-scale, for JET-like simulation.

On figure $6, \lambda_{n \text {,Eich }}$ is reported as a function of $D$. The estimate of the HD-model $\lambda_{\mathrm{HD}}$ for the simulation parameters and geometry is also reported with a blue dashed lines the comparison with the model will be discussed in the last section of this paper. We observe a perfect $\sqrt{D}$ scaling for purely diffusive simulations. The code is therefore robust in this low diffusion regime and is not dominated by spurious numerical diffusion. As the use of low diffusion coefficients are numerically challenging we have also proceeded to a test of convergence with the grid spatial resolution, and found that the estimate of $\lambda_{n}$ does not change when increasing the resolution of the grid in $\nabla B$-drift simulations.

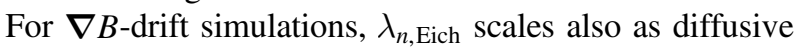
simulations for $D \geqslant 5 \times 10^{-3} \mathrm{~m}^{2} \mathrm{~s}^{-1}$, that is to say above $D_{\text {th }}$, which is in agreement with the result of section 3.1 where we have established that the diffusive operator is largely dominant in the transport in that range of $D$. This transport mechanism is thus the one setting the SOL width. This is followed by a stagnation of $\lambda_{n \text {,Eich }}$ corresponding to the transition toward the drift dominated regime, and $\lambda_{n \text {,Eich }}$ saturates at a non-zero value when $D$ tends towards zero. Note that the stagnation appears at $D<5 \times 10^{-3} \mathrm{~m}^{2} \mathrm{~s}^{-1}$, therefore at the same level of diffusion as the transition towards the drift dominated regime i.e. for $D<D_{\text {th }}$. Hence, at low diffusion, one indeed finds a regime where the $\nabla B$-drift is the mechanism entirely determining the SOL width. In such a regime, $\lambda_{n}$,Eich does not depend on the diffusion level. One can then define the SOL width associated with the $\nabla B$-drift large scale transport by $\lambda_{\nabla B}=\lim \lambda_{D \rightarrow 0}$. Here, we find $\lambda_{\nabla B} \approx 1.5 \mathrm{~mm}$.

The same scan can be made for $\lambda_{n \text {,int }}$ estimated at the outer mid-plane, that gives a similar trend but with a lower value of stagnation $\approx 1 \mathrm{~mm}$. Note that once again the $\lambda_{n \text {,int }}$ saturation appears at the same time as the supersonic transition, that is to say $D_{\text {th }} \sim D_{\text {trans }}$, confirming that the drift dominated regime is governed by a complex parallel equilibrium.

\subsection{Parametric dependencies}

In order to have further insight in the drift dominated regime, we now look at the parametric dependencies of the SOL width $\lambda_{\nabla B}$ in such a regime. The three scanned parameters are: the temperature $T_{e}=T_{i}=T$ for $T=25 \mathrm{eV}, 50 \mathrm{eV}, 75 \mathrm{eV}$, and $100 \mathrm{eV}$, and both poloidal and toroidal magnetic fields $B_{\mathrm{pol}}$ and $B_{\mathrm{tor}}$, which vary independently. Note that here the scaling is done for $\lambda_{n \text {,int }}$ because the target profiles are not well fitted with an Eich description. However, we have already assessed that the trend of $\lambda_{n, \text { Eich }}$ and $\lambda_{n, \text { int }}$ are similar.

In figure $7, \lambda_{n \text {,int }}$ are reported for the lowest diffusion value $D=2 \times 10^{-4} \mathrm{~m}^{2} \mathrm{~s}^{-1}$ as an estimate for $\lambda_{\nabla B}=$ $\lim _{D \rightarrow 0} \lambda_{\text {int }}$, as a function of the main parameters. We find that the SOL width depends on two principal parameters: the temperature and the poloidal magnetic field. First, $\lambda_{\nabla B}$ presents a positive correlation with $T$, of the form $T^{\alpha}$ with $\alpha$ strictly lower than $0.5\left(\lambda_{n \text {,int }} \propto \sqrt{T}\right.$ is indicated by the dashed lines). The scaling in $B_{\text {pol }}$ shows a decreasing dependency with $B_{\mathrm{pol}}$, close from the inverse dependency $\lambda_{n, \text { int }} \propto B_{\mathrm{pol}}^{-1}$ observed in experiment.

The SOL width results from a competition between the parallel and cross-field transport in the main SOL. Hence, one can define a fundamental SOL width associated with a mechanism by taking the equality between the parallel characteristic time $\left(\tau_{\|}\right)$and the characteristic time associated with this mechanism of cross-field transport $\left(\tau_{\perp}\right)$. We can thus get an estimate of the SOL width with:

$$
\tau_{\|} \sim \tau_{\perp} \quad \text { i.e } \quad \frac{v_{\|}}{L_{\|}} \sim \frac{v_{\nabla B}}{\lambda_{n}} .
$$

Using the following order of magnitude for each term, $v_{\nabla B} \sim \frac{T}{B R}, v_{\|} \sim c_{s} \propto \sqrt{T}$, and $L_{\|} \sim q R$, one gets:

$$
\lambda_{\nabla B} \sim \frac{T q R}{B R c_{S}} \propto \frac{a}{R} \sqrt{T} B_{\mathrm{pol}}^{-1} .
$$



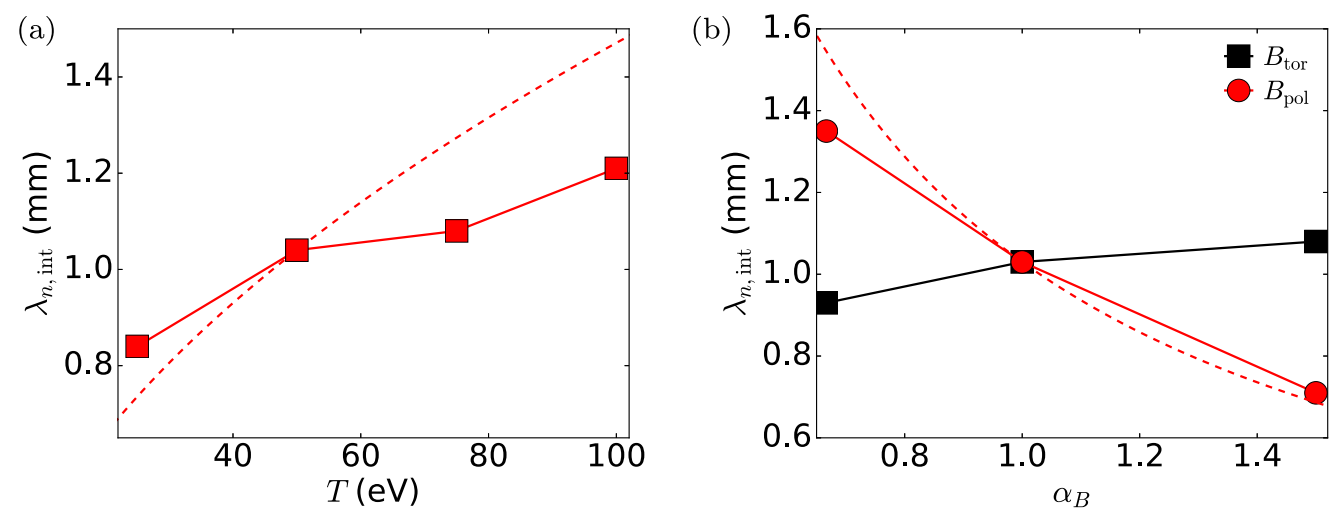

Figure 7. $\lambda_{n \text {,int }}$ SOL for $\nabla B$-drift simulations at $D=2 \times 10^{-4} \mathrm{~m}^{2} \mathrm{~s}^{-1}$ as a function of the temperature, with red dashed lines representing the $\sqrt{T}$ scaling (a), and as a function of the multiplicative factor, $\alpha_{B}$, on $B_{\text {pol }}$ and $B_{\text {tor }}$ with dashed lines representing the $B_{\text {pol }}^{-1}$ scaling (b).
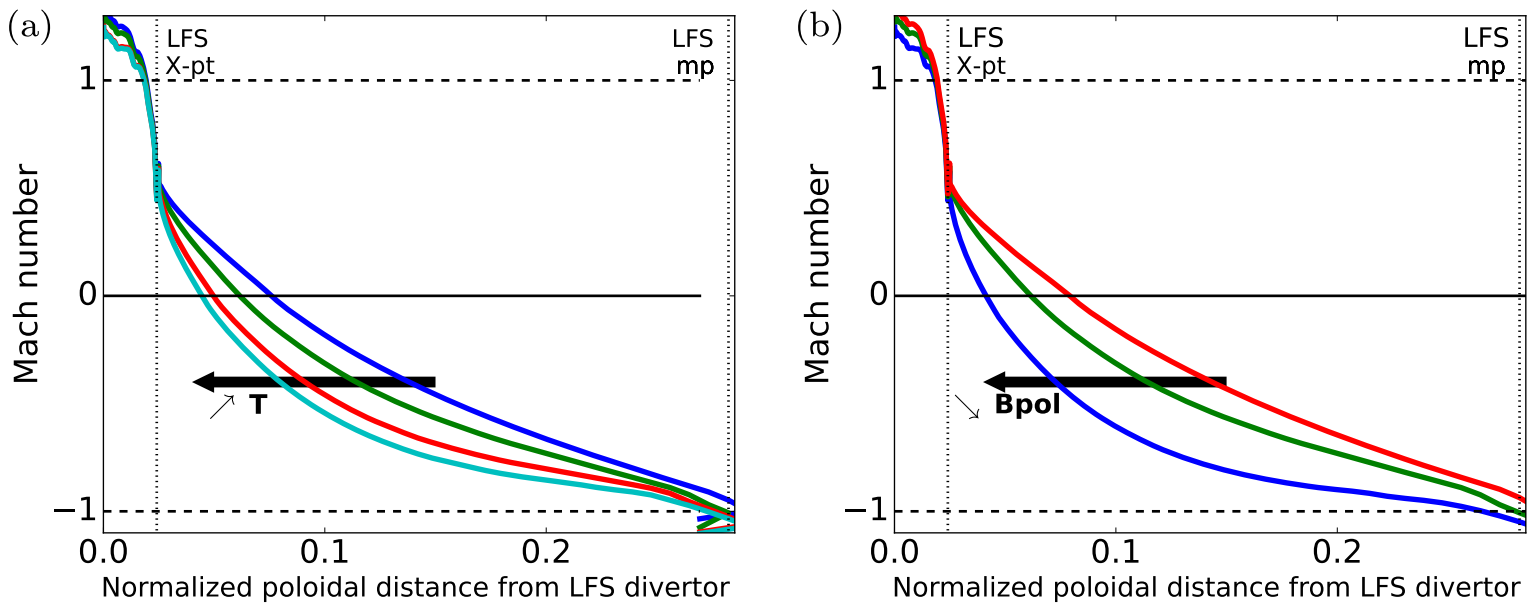

Figure 8. Mach number poloidal profile in the near SOL, for $\nabla B$-drift simulations for $D=2 \times 10^{-4} \mathrm{~m}^{2} \mathrm{~s}^{-1}$ for (a) several temperatures $T=25 \mathrm{eV}, 50 \mathrm{eV}, 75 \mathrm{eV}$, and $100 \mathrm{eV}$, respectively blue, green, red, and cyan lines, and (b) different $B_{\mathrm{pol}}=1 / 1.5,1,1.5 \times B_{\mathrm{pol}, 0}$ respectively blue, green, and red lines.

Note that the parametric dependencies of $\lambda_{\nabla B}$ in equation (12) are the ones found by the HD-model, as it follows similar lines of thought. The discrepancies of the numerical results with this rough estimation can be explained by the fact the parallel flows evolve with $B_{\text {pol }}$ and $T$, but this evolution is not captured by the simplified estimation. In fact, the estimation of $\lambda_{\nabla B}$ as the integrated radial displacement driven by the $\nabla B$-drift should depend on the parallel velocity and on the parallel length traveled by particles flowing toward the outer divertor target. On figure 8 , we observe that the stagnation point, separating particles flowing to the outer target and to the top of the machine, moves downward with an increase of $T$ or a decrease of $B_{\mathrm{pol}}$. This explains the weaker correlation between $\lambda_{\nabla B}$ and $T$ as the effective parallel length traveled by the particles decreases with $T$, which is not accounted for in the estimation of $\lambda_{\nabla B}$ scaling.

Finally, it is worth noting that there is an additive functional dependency with $B_{\text {tor }}$ weaker than the one in $B_{\mathrm{pol}}$, but not completely negligible either, this effect is also explainable by the parallel flows evolution.

\section{4. $\nabla$ B-drift dominated regime in other geometries}

The scan of the level of anomalous transport is also made for two other geometries: a circular limited and a COMPASS divertor magnetic equilibrium (figures 1(b) and (c)), to study whether the conclusions drawn in the previous sections are robust in other equilibriums and what the impact of the geometry is on the results. In this section, $\lambda_{n \text {,int }}$ at the outer mid-plane is used for the definition of the SOL width in order to compare the circular and the divertor geometry.

The same pattern is retrieved in both geometries, with two regimes depending on the level of anomalous transport: (1) a diffusive dominated regime at high $D$ where transport is dominated by diffusion and $\lambda_{n \text {,int }}$ scales as $\sqrt{D}$, and (2) a drift dominated regime with a stagnation of $\lambda_{n, \text { int }}$ and a non-zero limit when $D$ tends toward zero. Hence, the $\nabla B$-drift dominated regime exists independently of the magnetic configuration. Let us stress that the threshold value of $D, D_{\mathrm{th}}$ for the stagnation of $\lambda_{n, \text { int }}$, occurs in all cases at $D_{\text {trans }}$, that is to say the bifurcation towards supersonic flows. This reinforces the 


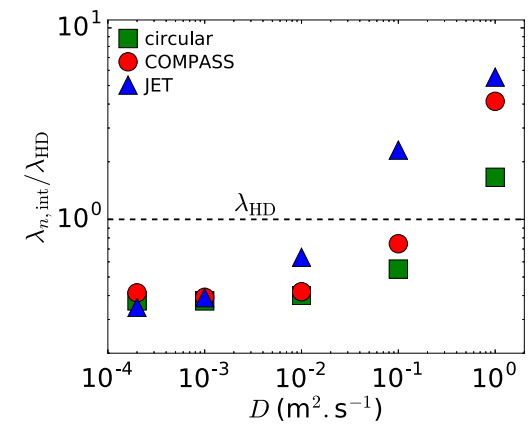

Figure 9. $\lambda_{n \text {,int }}$ for $\nabla B$-drift simulations normalized by the heuristic model prediction in JET-like (blue triangles), COMPASS-like (red circle) and circular geometries (green squares); both $x$ - and $y$-axis are in $\log$-scale.

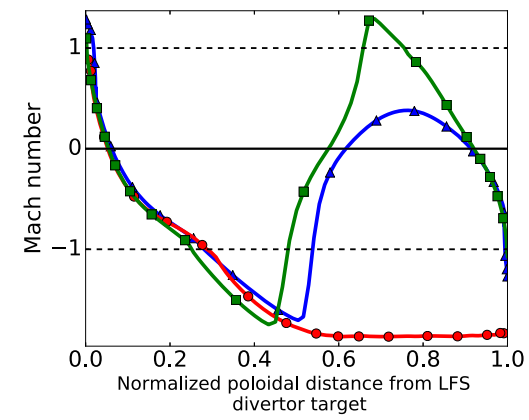

Figure 10. Mach number parallel profile for JET-like (blue with triangles), COMPASS-like (red line with circles) and circular (green line with squares) geometries at a distance of $\lambda_{n \text {,int }}$ from the separatrix and for $D=2 \times 10^{-4} \mathrm{~m}^{2} \mathrm{~s}^{-1}$.

conclusion that the supersonic flows are a characteristic inherent to the $\nabla B$-drift dominated regime, as they do not depend on the geometrical configuration. Furthermore, in both geometry $D_{\text {trans }}$ is correctly predicted by the simplified model presented in section 3.2 predicting $D_{\text {trans }}=$ $1.8 \times 10^{-1} \mathrm{~m}^{2} \mathrm{~s}^{-1}$ for circular geometry with a transition for $D$ between $1 \times 10^{-1} \mathrm{~m}^{2} \mathrm{~s}^{-1}$ and $5 \times 10^{-1} \mathrm{~m}^{2} \mathrm{~s}^{-1}$ in the simulations, and $D_{\text {trans }}=2.8 \times 10^{-1} \mathrm{~m}^{2} \mathrm{~s}^{-1}$ for COMPASS geometry with a transition for $D$ between $1 \times 10^{-1} \mathrm{~m}^{2} \mathrm{~s}^{-1}$ and $5 \times 10^{-1} \mathrm{~m}^{2} \mathrm{~s}^{-1}$ in the simulations.

We now compare the value of stagnation $\lambda_{\nabla B}$ with the HD-model estimate, that is to say an SOL width proportional to $\frac{a}{R} \sqrt{T} B_{\text {pol }}^{-1}$ also obtained in equation (12). We observe in figure 9(a) that the ratio $\lambda_{\nabla B} / \lambda_{\mathrm{HD}}$ is approximately constant at $\sim 2-3$ for the three geometries. This invariable ratio in the three geometries could be explained by the similarity of the parallel equilibrium. Indeed, in figure 10, reporting the Mach number poloidal SOL profile at a distance $\lambda_{n \text {,int }}$ from the separatrix, one can observe that the profiles at the low field side (LFS) coincide for the three geometries. This interpretation is consistent with the previous assertion that the parallel equilibrium has a strong impact on the establishment of $\lambda$ in this regime and that the discrepancy with the rough scaling law comes from the assumption of constant parallel speed and parallel length. This also explains that we find about the same ratio for all geometries for the same LFS parallel flows. At the high field side, the parallel flows differ greatly because of the symmetry breaking

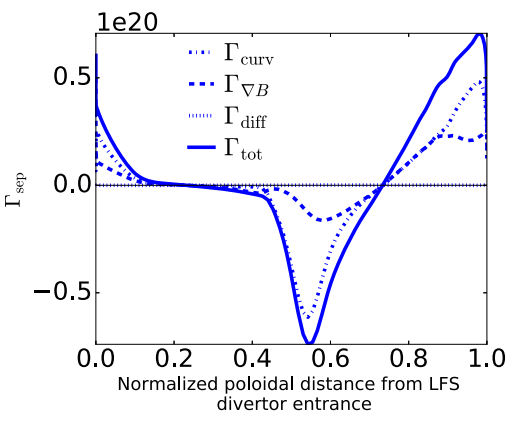

Figure 11. Poloidal profile of the local radial flux due to diffusion, $\nabla B$-drift, centrifugal drift, and total contribution for $D=2 \times$ $10^{-4} \mathrm{~m}^{2} \mathrm{~s}^{-1}$.

of divertor configuration: for COMPASS geometry the $\mathbf{u}_{\nabla B}$ contribution is weaker at the high field side (HFS) due to the triangularity implying weaker Pfirsch-Schlüter flows, on the contrary the circular geometry presents a relatively symmetric parallel profile.

\section{Influence of the centrifugal drift}

In fluid models, the centrifugal drift is often neglected under the assumption of a small Mach number in the SOL, $\frac{\left|\mathbf{u}_{\text {cent }}\right|}{\left|\mathbf{u}_{\nabla B}\right|}=M^{2} \ll 1$. However, we have previously assessed that in $\nabla B$-drift driven simulations supersonic flows are at play, thus neglecting $\mathbf{u}_{\text {cent }}$ is not relevant. In this section, the impact of the centrifugal drift on the transverse transport, parallel equilibrium and resulting SOL width is investigated in a JET-like magnetic configuration. For this purpose, we compare simulations including both $\nabla B$ and centrifugal drifts with the ones including only the $\nabla B$-drift.

In figure 11 we observe that the centrifugal drift flux is the largest term in the local particle flux crossing the separatrix whereas its flux-surface averaged contribution is negligible $\left\langle\Gamma_{\text {curv }}\right\rangle_{\mathrm{FS}} /\langle\Gamma \text { tot }\rangle_{\mathrm{FS}}<10 \%$. Contrary to the $\nabla B$-drift flux, the centrifugal drift flux is not only dependent on the density but also on the parallel flow amplitude. Now, the latter presents opposite asymmetry to the density. As a result the centrifugal drift has a negligible contribution to the particle flux averaged on a flux-surface. Nevertheless, $\mathbf{u}_{\text {cent }}$ has an indirect impact on the transverse transport, through the modification of the poloidal equilibrium and $\left.\left\langle\Gamma_{\nabla B \text {, withu }}\right\rangle_{\text {cent }}\right\rangle_{F S} \approx$ $1.25 \times\left\langle\Gamma_{\nabla B \text {, withoutu }}\right\rangle_{\mathrm{FS}}$. The plasma equilibrium response to the centrifugal drift is complex, and hard to capture as it results from a complex interplay between symmetry breaking of both the parallel velocity and the density, however several effects on the plasma equilibrium and the transverse transport can be underlined.

The first impact of $\mathbf{u}_{\text {cent }}$ is to stimulate the symmetry breaking. Poloidal profiles present greater variation for Mach number but also for $\lambda_{n \text {,int }}$ (figures 5 and 12) when it is included in the model. The amplitude of variation in the poloidal direction of $\lambda_{n \text {,int }}$ at LFS for simulation with centrifugal is twice as big as when $\mathbf{u}_{\text {cent }}$ is not included in the simulation, and the maximum of the Mach number is 1.5 


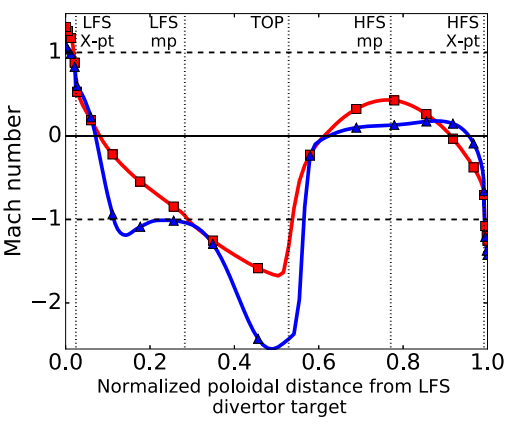

Figure 12. Poloidal profile of Mach number in the near SOL $\left(\frac{r}{a}=1.004\right)$ for $\nabla B$-drift simulation (red with square markers) and for $\nabla B$ and centrifugal drift simulation (blue with triangle markers) for $D=2 \times 10^{-4} \mathrm{~m}^{2} \mathrm{~s}^{-1}$.

times greater in the case with $\mathbf{u}_{\text {cent }}$. Moreover, $\mathbf{u}_{\text {cent }}$ favors the transition towards supersonic flows which appears at higher $D$ for simulations including $\mathbf{u}_{\mathrm{cent}}$.

This drift also has an impact on radial profiles. At $D<D_{\text {th }}$, radial profiles exhibit three distinct layers (figure 13(a)) in simulation including $\mathbf{u}_{\text {cent }}$. When moving from the separatrix to the far SOL, one first finds a boundary layer, of about $1.5 \mathrm{~mm}$ wide, characterized by a flat gradient, followed by a second layer with a steeper gradient and finally the last layer with an intermediate gradient. The first layer can be interpreted as a thin layer due to the radial displacement via drift convection of particles from the core. Simulations with only $\nabla B$-drift included also present the same kind of pattern, although less marked. The second layer is one where the transport would be essentially diffusive, in fact the e-folding length in this layer is about the same as the one in the simulations without diffusion. The explanation of the third layer is not straightforward, but it is most probably linked to the rise of the diffusion coefficient at the outer border of the mesh, or to the boundary condition at the wall. Note that this feature is also retrieved at the outer target, with irregular radial profiles presenting a flat layer in the area of the strike point.

\section{Impact of the inclusion of temperature variations and neutral species}

The previous model does not explicitly address either the heat transport or the neutral species. In this section, we look further at the impact of self-consistent electron and ion temperature variations on particles and power SOL width. Simulations are run for two values of thermal diffusivities $\chi_{\perp, i}=\chi_{\perp, e}=1 \mathrm{~m}^{2} \mathrm{~s}^{-1}$ and $\chi_{\perp, i}=\chi_{\perp, e}=0.1 \mathrm{~m}^{2} \mathrm{~s}^{-1}$, which are chosen as they are representative of the classical value of thermal conductivities in the pedestal used in mean field fluid simulations to fit the H-mode experiment [21-24]. Concerning the boundary conditions, the electron and ion temperatures at the inner boundary are taken as constant, equal to $100 \mathrm{eV}$. In figure 14, we observe that, for $1 \mathrm{~m}^{2} \mathrm{~s}^{-1}$, the value of $\lambda_{n \text {,int }}$ as a function of $D$ follows the same trend as in the isothermal simulation, and the value of saturation of $\lambda_{n \text {,int }}$ is similar to the one found in isothermal simulation $\lambda_{n \text {,int }}=1.1 \mathrm{~mm}$. Moreover, we find that $\lambda_{n, \text { int }} \approx \lambda_{q \text {,int }}$, under the assumption of high thermal diffusivity. Let us also underline that the equilibrium presents the same features as in isothermal simulations, that is to say the presence of supersonic flows and steady-state shock. In the set of anisothermal simulations, the supersonic transition occurs at higher $D$ due to a higher temperature at the separatrix. Simulations have been also run for this $\chi=0.1 \mathrm{~m}^{2} \mathrm{~s}^{-1}$. It is found that the value of $\lambda_{n \text {,int }}$ at low $D$ is not impacted, but as expected the value of stagnation of $\lambda_{q \text {,int }}$ is smaller, but only by about $15 \%$. In this range of $\chi$, the power SOL width is not strongly impacted due to the fact that $\lambda_{T} \gg \lambda_{n}$. Thus, in the $\nabla B$-drift dominated regime, and for the chosen thermal diffusivities, the assumption that $\lambda_{q} \approx \lambda_{n}$ seems reasonable, and the inclusion of temperature variation does not impact drastically the equilibrium.

Finally, we study the impact of the inclusion of neutral species in a low recycling regime in the case where only the $\nabla B$-drift is taken into account. Simulations have been run with tungsten PFC for the divertor target and beryllium PFC for the outer wall, with recycling coefficients of 0.99 and 1 , respectively. The density is set at $1 \times 10^{20} \mathrm{~m}^{-3}$ at the inner boundary, the density at the separatrix is about $1 \times 10^{20} \mathrm{~m}^{-3}$ in order to be in a low recycling regime (i.e. the framework of the HD-model), and a diffusion coefficient is taken to equal $2 \times 10^{-4} \mathrm{~m}^{2} \mathrm{~s}^{-1}$ in order to study the $\nabla B$ dominated regime. The inclusion of neutral species is expected to impact the results as it modifies the value of parallel velocity in the divertor region, and we have previously shown that the parallel equilibrium plays a major role in the establishment of the SOL width. In figure 15 we observe that the inclusion of neutral species results in a smaller amplitude of parallel flows, even if a supersonic transition is still reached in this case. This also impacts the parallel variation of density which is of a smaller amplitude and less abrupt. Looking at the profiles at the outer mid-plane, we observe that the simulation including neutral species presents radial profiles with a flatter gradient, resulting in a larger SOL width, $\lambda_{n, \text { int }}=1.7 \mathrm{~mm}$ and $\lambda_{q, \text { int }}=1.5 \mathrm{~mm}$. This can be explained by the fact that the Mach number amplitude is lower in the lower-outer quadrant, so that particles crossing the separatrix at the bottom and flowing toward the top of the machine are drifting for a longer time, resulting in a larger SOL width at the outer mid-plane. However, if we now look at the outer target profile we observe the inverse effect, where both density and heat flux profiles of simulation without the inclusion of neutral species are more spread than with their inclusion (figure 16). The detailed study of the physics in the divertor region is out of the scope of this paper, but let us mention several mechanisms that can explain this effect. First it is important to underline that the repartition of particle sources is completely modified, and the ionization of neutrals in the divertor region becomes a major player in the source for the plasma. This tends to decouple the physics of the main SOL and divertor, explaining that we observe opposite variation of the profile in 

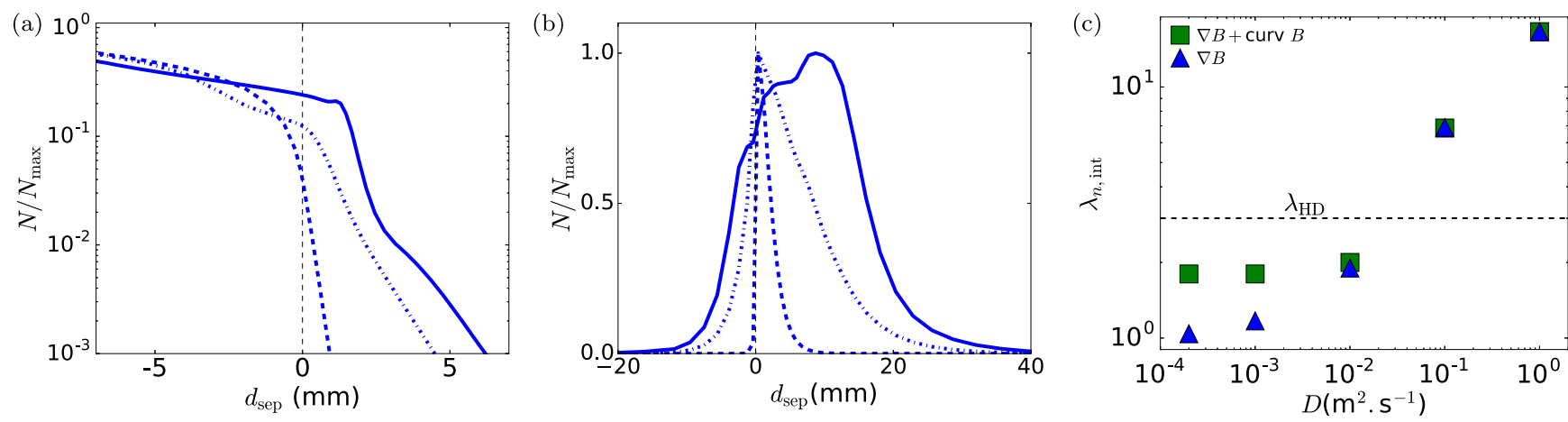

Figure 13. (a), (b) Density radial profile for diffusion (blue dashed lines), $\nabla B$-drift (blue dashed-dotted lines), and $\nabla B$ and centrifugal drift (blue full lines) simulations at the outer mid-plane and at the outer divertor (b) for $D=2 \times 10^{-4} \mathrm{~m}^{2} \mathrm{~s}^{-1}$. (c) $\lambda_{n \text {,int }} \operatorname{SOL}$ for $\nabla B$-drift simulations (blue triangles), and for $\nabla B$ and centrifugal drift simulations (green squares) as a function of the diffusion coefficient for JET-like simulation.

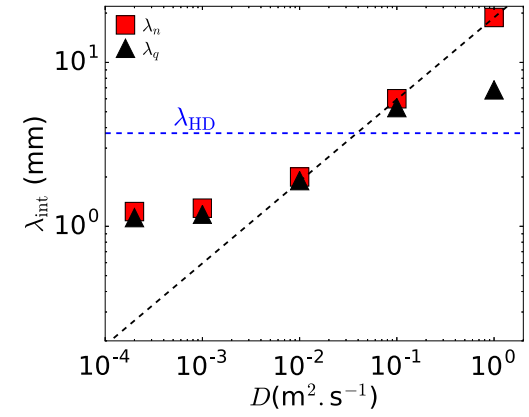

Figure 14. $\lambda_{n \text {,int }}$ and $\lambda_{q \text {,int }}$ for $\nabla B$-drift simulations as a function of the diffusion coefficient in anisothermal JET-like simulation.

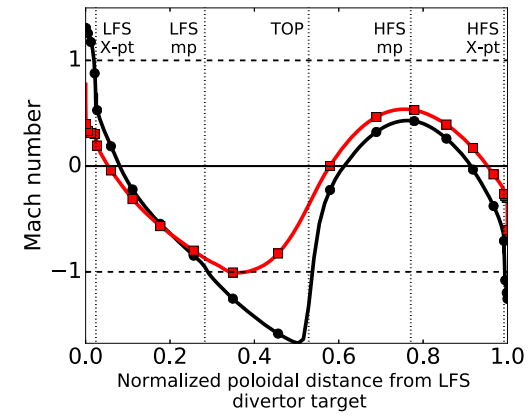

Figure 15. Mach number poloidal profile in the near SOL, for $\nabla B$-drift simulations for $D=2 \times 10^{-4} \mathrm{~m}^{2} \mathrm{~s}^{-1}$ for simulation without neutral species (red with square markers), and with neutral species (black with circle markers).

the two regions. This effect can also be observed in the parallel flow amplitude, in simulations without neutral species only a thin boundary layer at the vicinity of the separatrix presents a Mach number close to zero, when in simulation with neutral species the Mach number is close to zero at the divertor entrance on all the radial domains of the SOL. The fact the radial profile at the outer target presents a sharper gradient at the strike point where neutral species are included could be partly due to ionization. Indeed, this mechanism is proportional to the local density, so will be at a maximum at the strike point, this way reinforcing the plasma source at the peak location.

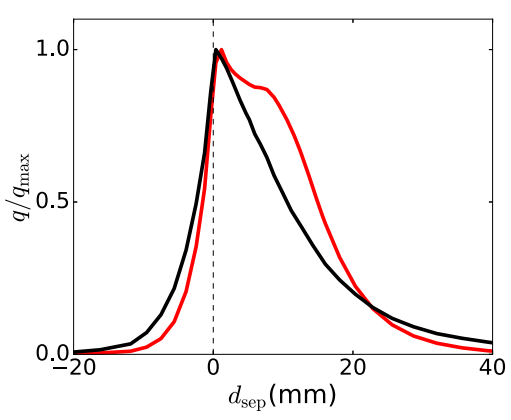

Figure 16. Heat flux radial profile $\nabla B$-drift simulations at the outer target for $D=2 \times 10^{-4} \mathrm{~m}^{2} \mathrm{~s}^{-1}$ for simulation without (red line) and with (black line) the inclusion of neutral species.

\section{Comparison of the results with the heuristic-drift based model and discussion on its hypothesis}

The reduced model used in section 3 corresponds to the main assumption made in the heuristic drift based model. Hence, it is worth comparing $\lambda_{n \text {,Eich }}$ with the one predicted by the HD-model, $\lambda_{\mathrm{HD}}=2 \sqrt{2} a \frac{B}{\left(B_{p} R\right)_{\mathrm{MP}}} \rho_{L}$. The numerical application for the JET geometry gives $\lambda_{\mathrm{HD}} \approx 3 \mathrm{~mm}$, namely twice the limit found in simulations $\lim _{D \rightarrow 0} \lambda_{n \text {,Eich }} \approx 1.5 \mathrm{~mm}$. Let us recall that this discrepancy between our numerical results and the HD-model estimate is also retrieved for the other geometries (section 4). Moreover, the functional dependency of $T$ $\left(\lambda_{\mathrm{HD}} \propto \sqrt{T}\right)$ is not retrieved in our simulations. The scaling in $B_{\mathrm{pol}}$, although it shows a better agreement with the model, is not unquestionable. Indeed, the dependency seems to be weaker than predicted, as highlighted by the lower $B_{\text {pol }}$ point, showing a variation $40 \%$ lower than the HD-model prediction (figure 7(b)).

The discrepancy between numerical results and the model is readily explainable by the parallel equilibrium, important in the establishment of the SOL width in the drift dominated regime. Indeed, the reduced model predicts $\lambda_{n \text {,Eich }}$ under the assumption that the parallel velocity is constant and equal to $c_{s} / 2$ in the lower-outer quadrant of the SOL, while the Mach number at low diffusion varies between $-c_{s}$ and $c_{s}$ in this poloidal extent (figure 3). Moreover, it assumes that particles flow from the outer target to the entry of the divertor when in 
low diffusion simulations the stagnation point is halfway between the outer mid-plane and the X-point so particles crossing the separatrix at the outer mid-plane flow to the top of the machine and not downward to the divertor target. In conclusion, within the main hypothesis of the model, the assumption of a $\nabla B$-drift dominated regime seems to lead to a contradiction with the first heuristic, that the parallel flow along $\mathrm{B}$ to the divertor competes at order unity with the usual Pfirsch-Schlüter parallel flow to the opposite side of the plasma' [11]. Hence, these results raise questions about the internal consistency of the HD-model, as the assumption $\nabla B$-drift as the main player of particle transport through the separatrix is in contradiction with the hypothesis on the parallel equilibrium. One should also keep in mind that some physics missing in the HD-model is proven to play an important role in the plasma equilibrium. First, the centrifugal drift is implicitly neglected in this model under the assumption that $u_{\|} / c_{s} \approx 0.5$. However, we have shown, on the contrary to this assumption, that the $\nabla B$-drift dominated regime is characterized by large amplitude flows and that $\mathbf{u}_{\text {cent }}$ plays a role in the establishment of the equilibrium (section 5). Furthermore, the HD-model explicitly addresses neither the heat transport nor the neutral species. If the assumption that the thermal transport is fast enough so that $\lambda_{n} \approx \lambda_{q}$ seems to be valid considering the classical diffusivity used in the pedestal, the impact of the neutral species should be taken into account as it impacts significantly both the equilibrium and the profiles.

The study of the charge balance is out of the scope of this work, and is a hard task from a numerical point of view due to the complexity of the equilibrium presenting stationary shock and sharp gradients. However, we know that the $\nabla B$ drifts are charge dependent, and in the $\nabla B$-drift dominated regime, the principal mechanism of transport leads to a charge separation and must therefore drives significant electric fields. Moreover, it has been shown that the electric drift has not only an importance in $\mathbf{E} \times \mathbf{B}$ turbulence but also drives large scale transport that needs to be taken into account even for a laminar approach [28]. Due to numerical constraints too demanding in the regime of low $D$ considered here, no simulation including the drift $\mathbf{E} \times \mathbf{B}$ has been run yet. However, one could expect a strong effect from the addition of the drift, especially in the low anomalous transport regime. Indeed, for a quasi-adiabatic electric field model, one has $\phi(\theta) \propto \ln N(\theta)$. Considering the large poloidal inhomogeneities of density, it would create a large $\partial_{\theta} \phi$, i.e. a large radial $\mathbf{E} \times \mathbf{B}$ drift velocity $u_{E}^{r}$. Such $\mathbf{E} \times \mathbf{B}$ flows could significantly modify the equilibrium.

\section{Conclusions}

In this work, we have studied the impact of the $\nabla B$-drift on the cross-field transport and on the SOL's width for different levels of anomalous transport using the fluid code SolEdge2D. The first conclusion of this work is that in all cases, including anisothermal simulations, simulations taking into account neutral species, and all geometries, a $\nabla B$-drift dominated regime is reached at low $D$. In this regime the value of SOL particles or power width is set by the magnetic drift and does not depend on
$D$. However, the relevance of this regime is disputable as it exists only at very low anomalous and collisional transport, well below the neoclassical level. This result is in agreement with the previous work of [15]. If we reach in all cases the $\nabla B$-drift dominated regime, the resulting SOL widths are significantly lower, by a factor of two to three, than the estimate of HD-model. Moreover, the parametric functional dependencies of the estimate, in particular the one on $T$, are not retrieved.

Another robust conclusion is that the $\nabla B$-drift dominated regime and the saturation of SOL width are linked to the transition toward a complex poloidal equilibrium, characterized by the apparition of supersonic flow and steady-state shock. This appearance of a supersonic transition is supported by a simple one dimensional analytical model, which has shown good agreement with numerical simulations. Moreover, the parallel equilibrium seems to play a role in the establishment of the SOL width, and it provides a reasonable explanation for the discrepancies between the estimate of the HD-model and the numerical results. Hence, a reduced model for the drift dominated regime should take into account the complexity of the parallel equilibrium. Furthermore, if at an intermediate and high diffusion level the role of centrifugal drift is not perceptible due to low amplitude of the Mach number, this is not the case in this regime characterized by large Mach number amplitude. In such a regime, the impact of the centrifugal drift should be explicitly taken into account, and we have shown in section 5 that the inclusion of this drift impacts significantly the equilibrium of the plasma.

Finally, one should discuss some open remaining questions. In this work we have not included the $\mathbf{E} \times \mathbf{B}$ drift, however it has been shown that the electric drift has not only an importance in $\mathbf{E} \times \mathbf{B}$ turbulence but also drives large scale transport that need to be taken into account, even for a laminar approach [28]. This is also the conclusion of [16], which shows that a drift dominated regime exists at a higher diffusion level only when considering the full drift dynamics, i.e. including the electric drift. Moreover, here the charge balance is not taken into account. Considering the large asymmetry at play in the simulations at low anomalous transport one could expect a large potential response, thus large $\mathbf{E} \times \mathbf{B}$ flows significantly modifying the equilibrium.

\section{ORCID iDs}

Camille Baudoin (10 https://orcid.org/0000-0001-5511-3959

Davide Galassi (iD https://orcid.org/0000-0003-3388-4538

Alberto Gallo (10) https://orcid.org/0000-0002-7472-7830

\section{References}

[1] Loarte A et al 2007 Power and particle control Nucl. Fusion 47 S203

[2] Eich T, Sieglin B, Scarabosio A, Fundamenski W, Goldston R J, Herrmann A and ASDEX Upgrade Team 2011 Inter-ELM power decay length for JET and ASDEX upgrade: measurement and comparison with heuristic driftbased model Phys. Rev. Lett. 107215001 
[3] Eich T et al 2013 Empiricial scaling of inter-ELM power widths in ASDEX upgrade and JET J. Nucl. Mater. 438 S72-7

[4] Thomas Eich et al 2013 Scaling of the tokamak near the scrape-off layer $\mathrm{H}$-mode power width and implications for ITER Nucl. Fusion 53093031

[5] Thornton A J et al 2014 Scaling of the scrape-off layer width during inter-ELM $\mathrm{H}$ modes on MAST as measured by infrared thermography Plasma Phys. Control. Fusion 56 055008

[6] Sun H J, Wolfrum E, Eich T, Kurzan B, Potzel S, Stroth U and ASDEX Upgrade Team 2015 Study of near scrape-off layer (SOL) temperature and density gradient lengths with Thomson scattering Plasma Phys. Control. Fusion 57 125011

[7] Scarabosio A et al 2013 Outer target heat fluxes and power decay length scaling in L-mode plasmas at JET and AUG J. Nucl. Mater. 438 S426-30

[8] Gunn J P et al Scrape-off layer power flux measurements in the Tore Supra tokamak J. Nucl. Mater. 438 S184-8

[9] Ricci P and Rogers B N 2009 Transport scaling in interchangedriven toroidal plasmas Phys. Plasmas 16062303

[10] Fedorczak N, Gunn J P, Nace N, Gallo A, Baudoin C, Bufferand H, Ciraolo G, Eich T, Ghendrih P and Tamain P 2017 Width of turbulent SOL in circular plasmas: a theoretical model validated on experiments in Tore Supra tokamak Nucl. Mater. Energy 12 838-43

[11] Goldston R J 2012 Heuristic drift-based model of the power scrape-off width in low-gas-puff H-mode tokamaks Nucl. Fusion 52013009

[12] Hinton F L and Hazeltine R D 1974 Kinetic theory of plasma scrape-off in a divertor tokamak Phys. Fluids 17 2236-40

[13] Faitsch M, Sieglin B, Eich T, Sun H J and Herrmann A 2015 Change of the scrape-off layer power width with the toroidal B-field direction in ASDEX Upgrade Plasma Phys. Control. Fusion 57075005

[14] Sieglin B, Eich T, Faitsch M, Herrmann A, Nille D, Scarabosio A and (ASDEX Upgrade Team) 2016 Density dependence of SOL power width in ASDEX Upgrade L-mode Nucl. Mater. Energy 12 216-20

[15] Reiser D and Eich T 2017 Drift-based scrape-off particle width in x-point geometry Nucl. Fusion 57046011
[16] Meier E T, Goldston R J, Kaveeva E G, Makowski M A, Mordijck S, Rozhansky V A, Senichenkov I Y and Voskoboynikov S P 2016 Analysis of drift effects on the tokamak power scrape-off width using SOLPS-ITER Plasma Phys. Control. Fusion 58125012

[17] Chankin A V and Coster D P 2013 The role of drifts in the plasma transport at the tokamak core-SOL interface $J$. Nucl. Mater. 438 S463-6

[18] Bufferand $\mathrm{H}$ et al 2015 Numerical modelling for divertor design of the WEST device with a focus on plasma-wall interactions Nucl. Fusion $\mathbf{5 5} 053025$

[19] Chankin A V 1997 Classical drifts in the tokamak SOL and divertor: models and experiment $J$. Nucl. Mater. 241 199-213

[20] Bufferand H et al 2017 Implementation of drift velocities and currents in SOLEDGE2D-EIRENE Nucl. Mater. Energy 12 $852-7$

[21] Chankin A V et al 2006 SOLPS modelling of ASDEX Upgrade H-mode plasma Plasma Phys. Control. Fusion 48839

[22] Horton L D et al 2005 Characterization of the H-mode edge barrier at ASDEX Upgrade Nucl. Fusion 45856

[23] Kallenbach A et al 2004 EDGE2D modelling of edge profiles obtained in JET diagnostic optimized configuration Plasma Phys. Control. Fusion 46431

[24] Gulejová B, Pitts R A, Wischmeier M, Behn R, Coster D, Horacek J and Marki J 2007 SOLPS5 modelling of the type III ELMing H-mode on TCV J. Nucl. Mater. 363 1037-43

[25] Fundamenski W et al 2007 Dissipative processes in interchange driven scrape-off layer turbulence Nucl. Fusion 47417

[26] Ghendrih P et al 2011 Transition to supersonic flows in the edge plasma Plasma Phys. Control. Fusion 53054019

[27] Bufferand H, Ciraolo G, Dif-Pradalier G, Ghendrih P, Tamain P, Marandet Y and Serre E 2014 Magnetic geometry and particle source drive of supersonic divertor regimes Plasma Phys. Control. Fusion 56122001

[28] Galassi D, Tamain P, Bufferand H, Ciraolo G, Ghendrih P, Baudoin C, Colin C, Fedorczak N, Nace N and Serre E 2017 Drive of parallel flows by turbulence and large-scale $E \times B$ transverse transport in divertor geometry Nucl. Fusion $\mathbf{5 7}$ 036029 